determine the nature of the vector field representing the wind structure.

ARnold Dresden, Secretary of the Chicago Section.

\title{
THE APRIL MEETING OF THE SAN FRANCISCO SECTION
}

The thirty-fifth regular meeting of the San Francisco Section was held at Stanford University on Saturday, April 10. The chairman, Professor Blichfeldt, presided. The total attendance was twenty-three, including the following thirteen members of the Society:

Professor R. E. Allardice, Professor B. A. Bernstein, Professor H. F. Blichfeldt, Professor Thomas Buck, Professor Florian Cajori, Professor M. W. Haskell, Professor L. M. Hoskins, Professor D. N. Lehmer, Professor W. A. Manning, Professor H. C. Moreno, Dr. F. R. Morris, Professor C. A. Noble, Dr. Pauline Sperry.

The action of the executive committee in making plans for a meeting of the Section at the University of Washington June 17-19, in connection with the Pacific division of the American association for the advancement of science was approved. It was decided that this should be a special meeting of the Section, the regular Fall meeting to take place October 23, 1920, as scheduled.

The following papers were presented and discussed:

(1) Professor H. F. Blichfeldt: "On the approximate representation of irrational numbers" (preliminary report).

(2) Professor Florian Cajori: "Note on the history of divergent series."

(3) Professor L. M. Hoskins: "Note on the Lorentz transformation and the notion of time."

(4) Professor E. T. BeLL: "The twelve elliptic functions related to sixteen doubly periodic functions of the second kind."

(5) Professor E. T. BeLl: "Certain remarkable sums related to 3,5 , and 7 squares."

(6) Professor E. T. BeLL: "Parametric solutions for a fundamental equation in the general theory of relativity." 
In the absence of the author, Professor Bell's papers were read by title. Abstracts of the papers follow below.

1. The theory of approximate solutions, in terms of integers, of linear equations with irrational coefficients, has been developed mainly by Kronecker and Minkowski. Thus, the equations $x_{i}-a_{i} x_{n}=0(i=1,2, \cdots, n-1)$ possess an infinite number of sets of integral solutions with errors $\epsilon_{i}<$ $\frac{n-1}{n} \cdot \frac{1}{x_{n}^{1 /(n-1)}}$. Kronecker has shown that the equations $x_{i}-a_{i} x_{n}=b_{i}(i=1,2, \cdots, n-1)$ possess an infinite number of sets of integral solutions, with errors $\epsilon_{i}$ at the outset assigned as small as we please, provided no equation exists of the form $\sum_{i} k_{i} a_{i}=k_{n}$, where the $k_{i}, k_{n}$ are integers. Professor Blichfeldt finds that we cannot in general assume $\epsilon_{i}$ $<N x_{n}{ }^{-1 /(n-1)}$, where $N$ is any given constant.

2. Professor Cajori pointed out that, as early as 1843, A. De Morgan advanced a summation formula for divergent series, discussed asymptotic series and used a principle relating to divergent series; these researches entitle him to the rank of a pioneer in the development of the modern theory of divergent series.

3. Professor Hoskins called attention to an apparent paradox resulting from Einstein's interpretation of the Lorentz transformation. If two exactly similar clocks $C$ and $C^{\prime}$ keep time correctly for a system $S$ while both are at rest at a point $A$ of the system, and if $C$ remains at $A$ while $C^{\prime}$ is carried in any closed path with uniform speed $v$ and brought back to $A$, then according to Einstein the elapsed time as indicated by $C^{\prime}$ will be less than that indicated by $C$, the relation being approximately $T^{\prime}=T\left[1-1 / 2(v / V)^{2}\right]$. It is, however, equally legitimate to refer the motion to a coordinate system with respect to which $C^{\prime}$ remains stationary while $C$ describes a closed path with uniform speed $v$, and from this point of view the reasoning of Einstein leads to the conclusion that $T=$ $T^{\prime}\left[1-1 / 2(v / V)^{2}\right]$. There seems no ground for regarding the reasoning as valid in one case and not in the other. Is it not possible that in both cases it is based upon an illegitimate physical interpretation of the Lorentz transformation? 
4. The twelve elliptic functions considered by Glaisher and other writers are shown in Professor Bell's paper to arise as degenerate forms from a set of sixteen doubly periodic functions of the second kind which may be generated by the operations of a certain group from any one of them. The same functions give also, by differentiation, transformations of the second order, etc., all of the doubly periodic functions of the third kind considered by Hermite, Biehler, Appell, and others, with many more. The paper will appear in the Messenger of Mathematics.

5. In connection with his recent determinations of the numbers of representations of integers as sums of 3,5,7, 9, 11, and 13 squares, (which will appear in the American Journal), Professor Bell remarked that incidentally the 3-square results yield the class number by finite processes only. In this paper new binary class number formulas and analogous results related to quinary and senary forms are derived.

6. The equation solved in Professor Bell's note is that for the square of the line element in the general non-euclidean time-space, which holds a central position in Einstein's theory of gravitation. The solution is not free from quadratures. It is shown that particular solutions of certain other equations in general dynamics may be similarly found. The note will appear in the Philosophical Magazine.

B. A. Bernstein, Secretary of the Section.

\section{THE SUM OF THE FACE ANGLES OF CERTAIN POLYHEDRONS IN $n$-SPACE.}

BY DR. HARRIS F. MACNEISH.

(Read before the American Mathematical Society December 30, 1919.)

A SIMPLE polyhedron in $n$-space is defined as a set of $r$ spaces ${ }_{n-i} C_{r-i}$ of which intersect in each $i$-space; $r>i$; $r=1,2, \cdots, n-1 ; i=0,1, \cdots, n-2$.

For a simple polyhedron $P^{n}$ in $n$-space we denote the sum of the angles of all the plane faces by $S_{p n}$, the number of 